\title{
Search for long-lived neutral particles decaying into lepton-jets with the ATLAS detector in proton-proton collision data at $\sqrt{s}=13 \mathrm{TeV}$
}

\author{
Antonio Policicchio*t \\ Universitá della Calabria and INFN-CS \\ E-mail: antonio.policicchiodcern.ch
}

Several models of elementary particle physics beyond the Standard Model, predict the existence of neutral particles that can also be long lived and decay in collimated jets of light leptons and hadrons (lepton-jets). The search for displaced lepton-jets in a $3.4 \mathrm{fb}^{-1}$ proton-proton collision data sample recorded by ATLAS at $\sqrt{s}=13 \mathrm{TeV}$ during 2015 data taking period is summarized. The selected events are compared with the Standard Model expectations and with BSM predictions.

38th International Conference on High Energy Physics

3-10 August 2016

Chicago, USA

*Speaker.

${ }^{\dagger}$ On behalf of the ATLAS Collaboration. 


\section{Introduction}

Several possible extensions of the Standard Model (SM) predict the existence of a dark sector that is weakly coupled to the visible one [1]. Depending on the structure of the dark sector and its coupling to the SM, some unstable dark states may be produced at colliders and decay back to SM particles with sizeable branching fractions. An extensively studied case is one in which the two sectors couple via the vector portal, where a dark photon $\left(\gamma_{\mathrm{d}}\right)$ with mass in the $\mathrm{MeV}$ to $\mathrm{GeV}$ range mixes kinetically with the SM photon [2]. If the $\gamma_{\mathrm{d}}$ is the lightest state in the dark sector, it will decay to SM particles, mainly to leptons and possibly light mesons. Due to its weak interactions with the SM, it can have a non-negligible lifetime. At the LHC, these dark photons would typically be produced with large boost resulting in collimated jet-like structures containing pairs of leptons and/or light hadrons (lepton-jets, LJs). If produced away from the interaction point (IP), they are referred to as "displaced LJs". The search described in the present contribution looks for events with a pair of displaced LJs originating from the decay of an heavy particle and the LJ constituents are electrons, muons, and pions. The search employs the full dataset collected by ATLAS [3] during 2015 at $\sqrt{s}=13 \mathrm{TeV}$, corresponding to an integrated luminosity of $3.4 \mathrm{fb}^{-1}$ [4].

\section{Lepton-jets reconstruction and selection}

The search adopts a generic definition of $\mathrm{LJ}$ in order to make the analysis as model-independent as possible. The LJ contains at least one $\gamma_{d}$ decaying far from the primary vertex. The LJs are thus expected highly isolated in the inner tracker. Three topologies of LJs are considered: TYPE0, TYPE1 and TYPE2. A TYPE0 LJ is a cluster of at least two muons identified into the muon spectrometer with a veto on jets in a cone opening $\Delta R=0.5$. It is the signature of a $\mathrm{LJ}$ with all $\gamma_{d}$ decaying to muon pairs. A TYPE1 LJ is a cluster of at least two muons and jets in a cone opening $\Delta R=0.5$. It is the signature of at least one $\gamma_{d}$ decaying to a muon pair and at least another one decaying to an electron or pion pair. A TYPE2 LJ is a jet with low electromagnetic fraction and no muons in a cone opening $\Delta R=0.5$. It is the signature of a $\mathrm{LJ}$ dark photons decaying to electron/pion pairs. The triggers used to select displaced LJs of TYPE0 and TYPE1 are multi-muon triggers with muons reconstructed only in the muon spectrometer. For the TYPE2 LJs the single jet trigger with low electromagnetic fraction is used: only LJs produced in the hadronic calorimeters are selected, reducing the background due to the QCD jets. The events that pass the trigger filters have to satisfy additional requirements to separate the signal from the background. Two major sources of background are expected: muons from cosmic rays and QCD multi-jet events. The cosmic-ray events are reduced using jet timing, and for muons there is an additional requirement: the perigee to the beam line of the muon spectrometer track has to be close to the primary vertex of the event. QCD multi-jet background is reduced using track isolation in the inner tracker, jet electromagnetic fraction and width of the jets. Two additional requirements are used: exactly two reconstructed LJs in the event; the absolute value of the azimuthal angle $\Delta \phi$ between the two LJs must be $\geq 1$ (i.e. in the geometric limits of the LJ reconstruction cone). The residual QCD multi-jet background and cosmics background is evaluated using a data-driven matrix ABCD method. Details on the selection and background estimation can be found in [4]. 


\section{Results}

Table 1 shows the final result: the observed data events in the signal region, and the expected multijet and cosmic-ray residual contaminations. Taking into account the expected high background for the TYPE2-TYPE2 events, the ABCD method is performed on three categories: all events, excluding the TYPE2-TYPE2 events, and only the TYPE2-TYPE2 events. In all categories, no evidence of signal is observed.

Table 1: Results of the ABCD compared with the observed events on data. Table taken from [4].

\begin{tabular}{|c|c|c|}
\hline Category & Observed events & Expected background \\
\hline \hline All events & 285 & $231 \pm 12$ (stat) \pm 62 (syst) \\
\hline TYPE2-TYPE2 excluded & 46 & $31.8 \pm 3.8($ stat $) \pm 8.6($ syst $)$ \\
\hline TYPE2-TYPE2 only & 239 & $241 \pm 41$ (stat) \pm 65 (syst) \\
\hline
\end{tabular}

In the absence of a signal, the results of the search for LJ production are used to set upper limits on the product of cross section and Higgs decay branching fraction to LJs, as a function of the $\gamma_{\mathrm{d}}$ mean lifetime in the two Falkowski-Ruderman-Volansky-Zupan (FRVZ) models [2] which predict non-SM Higgs boson decays to LJs. As shown in Figure 1, the first FRVZ model produces two $\gamma_{\mathrm{d}}$ 's while the second produces four $\gamma_{\mathrm{d}}$ 's. In the first model (left), the dark fermion decays to a $\gamma_{\mathrm{d}}$ and a lighter dark fermion $f_{\mathrm{d}_{1}}$, assumed to be the HLSP (Hidden Lightest Stable Particle). In the second model (right), the dark fermion $f_{\mathrm{d}_{2}}$ decays to an HLSP and a dark scalar $s_{\mathrm{d}_{1}}$ that in turn decays to pairs of dark photons. The mass of the dark photon is assumed $400 \mathrm{MeV}$. Details on the models are in [4]. The $\gamma_{\mathrm{d}}$ decay lifetime, $\tau$ (expressed as $\tau$ times the speed of light $c$ ) is
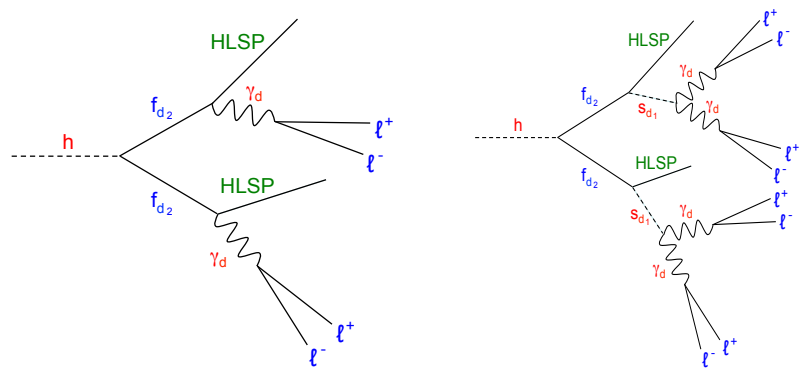

Figure 1: The two FRVZ models used as benchmarks in the analysis. Figure taken from [4].

a free parameter of the model. The $C L s$ method is used to determine the exclusion limits, where the signal region is populated from the data-driven background estimate and from the appropriate signal hypothesis. The resulting exclusion limits on the $\sigma \times \mathrm{BR}$, assuming the $125 \mathrm{GeV}$ Higgs boson SM gluon-fusion production cross section $\sigma_{\mathrm{SM}}=44.13 \mathrm{pb}$ are shown in Figure 2 as a function of the $\gamma_{\mathrm{d}}$ mean lifetime for the Higgs $\rightarrow 2 \gamma_{\mathrm{d}}+X$ and Higgs $\rightarrow 4 \gamma_{\mathrm{d}}+X$ models. TYPE2-TYPE2 events are excluded from the limit evaluation. The expected limit is shown as the dashed curve and the solid curve shows the observed limit. The horizontal lines correspond to $\sigma \times \mathrm{BR}$ for two values of the BR of the Higgs boson decay to dark photons. The same exclusion limits have been derived for the $800 \mathrm{GeV}$ heavy scalar. Table 2 shows the ranges in which the $\gamma_{\mathrm{d}}$ lifetime $(\mathrm{c} \tau)$ is excluded at the $95 \% \mathrm{CL}$ for the $m_{H}=125 \mathrm{GeV}$ Higgs $\rightarrow 2 \gamma_{\mathrm{d}}+X$ and Higgs $\rightarrow 4 \gamma_{\mathrm{d}}+X$, assuming a BR 

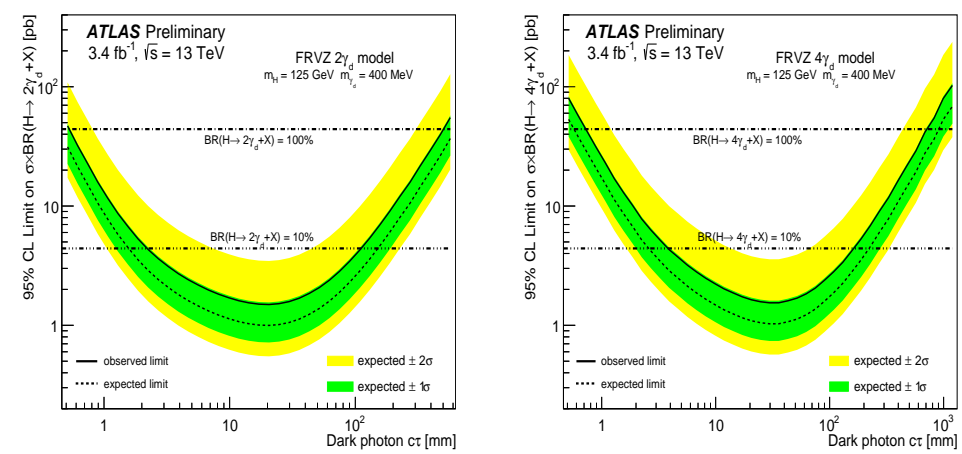

Figure 2: The $95 \%$ upper limits on the $\sigma \times \mathrm{BR}$ for the FRVZ $125 \mathrm{GeV} \mathrm{Higgs} \rightarrow 2 \gamma_{\mathrm{d}}+X$ (left) and Higgs $\rightarrow 4 \gamma_{\mathrm{d}}+X$ (right) benchmark models as a function of the $\gamma_{\mathrm{d}}$ lifetime (c $\tau$ ). The horizontal lines correspond to $\sigma \times \mathrm{BR}$ for two values of the BR of the Higgs boson decay to dark photons. TYPE2-TYPE2 events are excluded. Figure taken from [4].

of $10 \%$. It also shows the $95 \% \mathrm{CL} c \tau$ exclusion ranges for the $m_{H}=800 \mathrm{GeV}$, assuming a $5 \mathrm{pb}$ production cross section and a $100 \%$ BR to $\gamma_{\mathrm{d}}$.

Table 2: Ranges of $\gamma_{\mathrm{d}}$ lifetime (c $\tau$ ) excluded at $95 \%$ CL for Higgs $\rightarrow 2 \gamma_{\mathrm{d}}+X$ and Higgs $\rightarrow 4 \gamma_{\mathrm{d}}+X$, assuming for the $125 \mathrm{GeV}$ Higgs a $10 \%$ BR and the Higgs boson SM gluon fusion production cross section, and for the $800 \mathrm{GeV}$ Higgs-like scalar a $\sigma \times \mathrm{BR}=5 \mathrm{pb}$. TYPE2-TYPE2 events are excluded. Table taken from [4].

\begin{tabular}{|c|c|c|}
\hline FRVZ model & $m_{\mathrm{H}}(\mathrm{GeV})$ & Excluded $\mathrm{c} \tau[\mathrm{mm}]$ \\
\hline Higgs $\rightarrow 2 \gamma_{\mathrm{d}}+X$ & 125 & $2.2 \leq \mathrm{c} \tau \leq 111.3$ \\
\hline Higgs $\rightarrow 4 \gamma_{\mathrm{d}}+X$ & 800 & $3.8 \leq \mathrm{c} \tau \leq 163.0$ \\
\hline Higgs $\rightarrow 2 \gamma_{\mathrm{d}}+X$ & 125 & $0.6 \leq \mathrm{c} \tau \leq 63$ \\
\hline Higgs $\rightarrow 4 \gamma_{\mathrm{d}}+X$ & 800 & $0.8 \leq \mathrm{c} \tau \leq 186$ \\
\hline
\end{tabular}

A recently observed anomalous internal pair creation in Beryllium- $8\left({ }^{8} \mathrm{Be}\right)$ is interpreted as a possible signature of a light, neutral "protophobic" boson of $16.7 \mathrm{MeV}$, decaying to electron pairs from the dark sector [5]. TYPE2-TYPE2 topology can be used to put limits on the lifetime in the contest of this "protophobic" mode assuming a $100 \% 800 \mathrm{GeV}$ heavy scalar decay to a $\gamma_{\mathrm{d}}$ of 16.7 $\mathrm{MeV}$ mass, decaying only to electron pairs (the detection and reconstruction efficiencies for the $\gamma_{\mathrm{d}}$ decay in the HCAL are almost independent from the $\gamma_{\mathrm{d}}$ mass). The 95\% CLs method gives a $c \tau$ exclusion limit $2.7 \mathrm{~mm} \leq c \tau \leq 21 \mathrm{~mm}$, assuming a reference $\sigma \times \mathrm{BR}$ of $10 \mathrm{pb}$ [4].

\section{References}

[1] M. J. Strassler and K. M. Zurek, Phys. Lett. B 651374 (2007)

[2] A. Falkowski, J. T. Ruderman, T. Volansky and J. Zupan, JHEP 05077 (2010)

[3] The ATLAS Collaboration, JINST 3, S08003 (2008)

[4] The ATLAS Collaboration, ATLAS-CONF-2016-042 (2016)

[5] A. J. Krasznahorkay et al., Phys. Rev. Lett. 116 (2016) 042501 